

\title{
Neutron yield measurements on a TMX endplug
}

\author{
D. R. Slaughter
}

Manuscript date: March 17, 1980

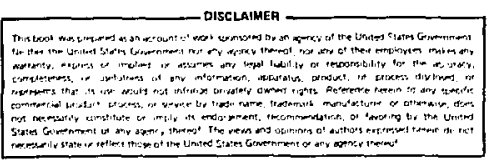

\section{LAWRENCE LIVERMORE LABORATORY University of California $\bullet$ Livermore, Cäif Jurnia $\bullet 94550$}




\section{CONTENTS}

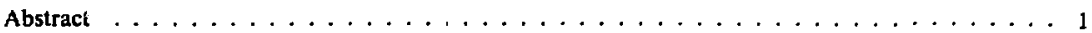

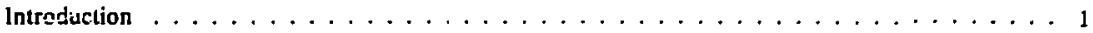

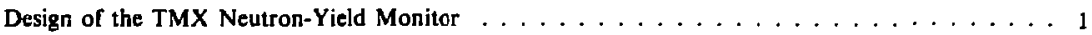

Calibration .............................. . . . . 4

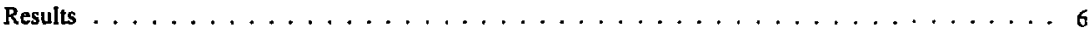

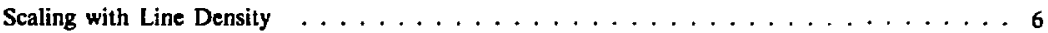

Scaling with Plasma Diamagnetism $\ldots \ldots \ldots \ldots \ldots \ldots$

Scaling with Neutral Beam Current $\ldots \ldots \ldots \ldots \ldots \ldots$

Decay of the Fusion Rate Following Beam Shutoff $\ldots \ldots \ldots \ldots \ldots$

Estimating Mean Ion Energy $\overline{\mathbf{w}}_{\mathrm{i}} \ldots \ldots \ldots \ldots \ldots \ldots \ldots \ldots \ldots$

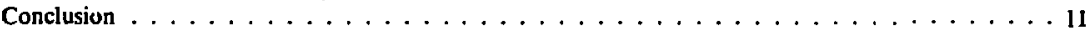

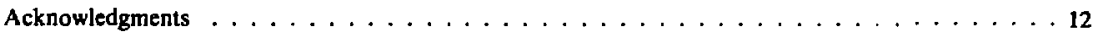

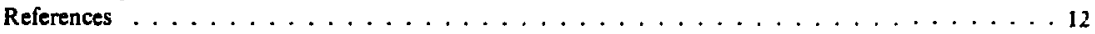




\section{Neutron yield measurements on a TMX endplug}

\section{ABSTRACT}

Neutron yield measurements were made on the east endplug of TMX using a calibrated recoil proton counter. The detector consists of a liquid scintillator (NE 213) with a pulse shape discrimination property that allows for identifying photon and neutron interactions. An energy threshold is established to suppress the response to scattered neutrons with energies lower than 1 to $2 \mathrm{MeV}$. Results indicate there are typical neutron yields of 2 to $3 \times 10^{11} \mathrm{n} / \mathrm{s}$ during a $25-\mathrm{ms}$ discharge with $200 \mathrm{~A}$ of $20-\mathrm{keV}$ neutral beam injection into the endplug.

\section{INTRODUCTION}

The Tandem Mirror Experiment' (TMX) at Lawrence Livermore Laboratory (LLL) produces magnetically confined plasmas of relatively high ion energy. Plasma is confined radially by a long solenoid $(L=5.5 \mathrm{~m})$ that produces an axial magnetic field in the range 0.05 to $0.2 \mathrm{~T}$. Axial confinement is achieved by electrostatically plugging the ends by the positive potential developed in a mirror-confined plasma. The endplugs are similar to the 2XIIB magnetic mirrors. Most shots consist of deuterium plasma at densities near $10^{13} \mathrm{~cm}^{-3}$ in the solenoid and $\sim 3 \times 10^{13} \mathrm{~cm}^{-3}$ in the endplugs. Heating and refueling is accomplished by injecting about $200 \mathrm{~A}$ of neutral deuterium into each endplug at nominally $20 \mathrm{keV}$ ( $12 \mathrm{keV}$ mean energy) as well as 10 to 50 amps of deuterium at nominally $30 \mathrm{keV}$ (20 keV mean energy). Fusion of deuterium (D-D) occurs principally in the endplug regions as a result of reactions among hot ions as well as interactions between the injected beam and hot or cold deuterium in the plasma.

D-D fusion reactions proceed according to either of the two branches shown below.

$$
\begin{aligned}
& \mathrm{D}+\mathrm{D} \% \\
& 48 \% \mathrm{p}(2.45 \mathrm{MeV})+{ }_{2}^{3} \mathrm{He}(0.82 \mathrm{MeV}) .
\end{aligned}
$$

These reactions occur with approximately equal frequency. The branching ratios are from Miley et al. ${ }^{2}$ for Maxwellian plasma at $\bar{w}_{i}=11 \mathrm{keV}$. The fusion rate in the machine can be determined from the generation rate of $2.45-\mathrm{MeV}$ neutrons; the latter is helpful in determining machine power balance. It may also help in making an estimate of the mean ion energy if the plasma volume and density are known, because the D-D fusion cross section is very sensitive to the energy of reacting ions.

\section{DESIGN OF THE TMX NEUTRON-YIELD MONITOR}

A neutron monitor was installed near the east endplug of TMX to measure the fusion rate in the plug. It is located outside the vacuum vessel, $3.3 \mathrm{~m}$ below the center (Fig. 1). Although there is an unobstructed view of the plasma through the magnet structure, the data reported here were obtained during a period in which there was significant attenuation in a 25-mm-thick (0.75-mean-free-paths) diagnostic port window and the 10 -mm-thick (0.3mean-free-paths) magnetic and electromagnetic shielding around the monitor. Within the shielding there are two recoil proton spectrometers (Fig. 2).

A liquid organic scintillation detector (NE213) was chosen because of its high hydrogen density $\left(4.8 \times 10^{22} \mathrm{~cm}^{-3}\right)$ and, consequently, high efficiency. In addition, its fluorescence is slower for 


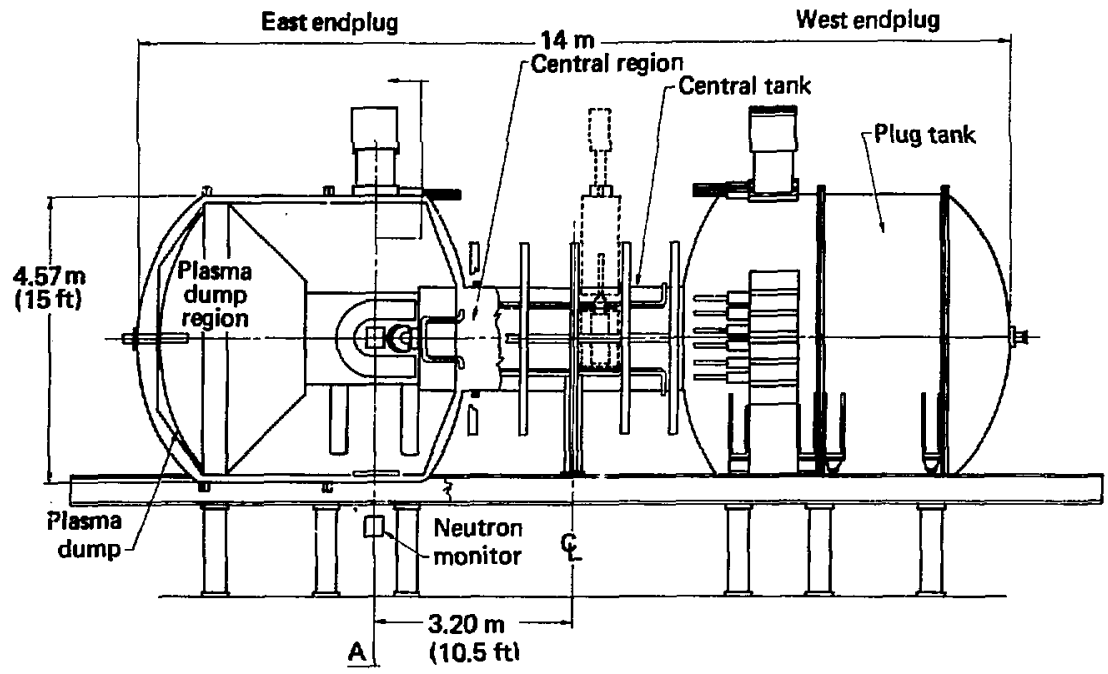

FIG. 1. Tanden Mirror Experinent (TMX) at Lawrence Livermore Laboratory. The TMX neutron yield monitor is located $3.3 \mathrm{~m}$ below the east endplag.

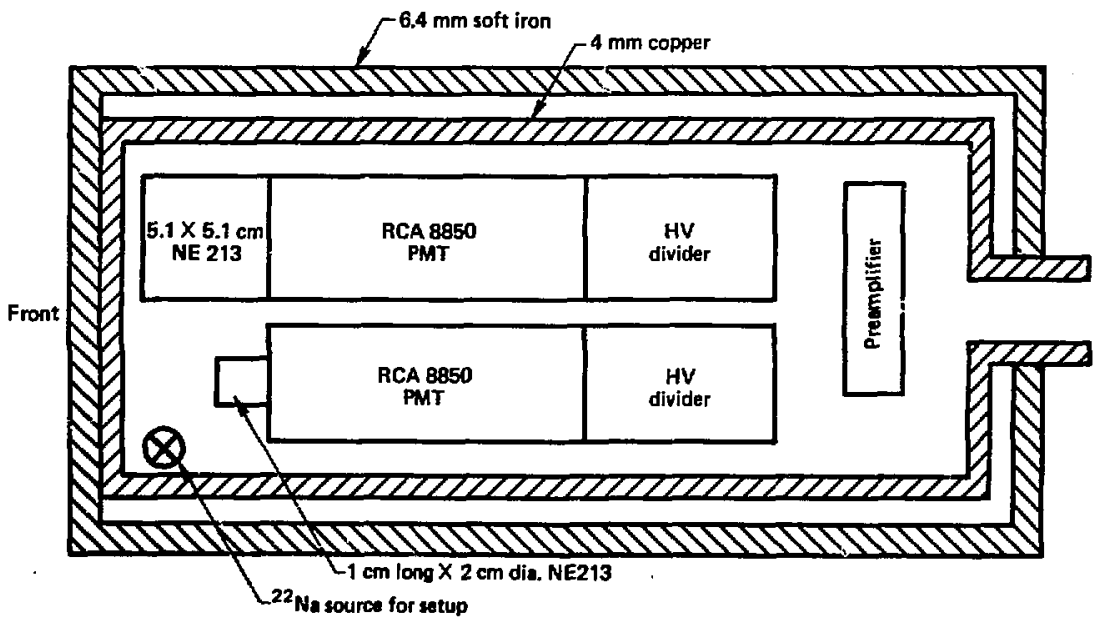

FIG. 2. In a TMX newtrew mowitor, the nentron detectors are ewclosed within a copper if shield and a solt irow

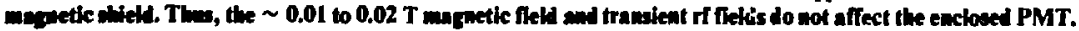


recoil protons 3,4 than for photoelectrons. This means that pulse-shape discrimination may be used to identify neutron and photon interactions in the detector, so that only the former are recorded. Finally, the scintillation light output is a weil known function of recoil proton energy ${ }^{5}$; consequently, a pulse height spectrum of the light output may be unfolded to determine the incident neutron energy spectrum. ${ }^{6.7}$

A schematic of the detector and electronics is shown in Fig. 3. Each scintillator is optically coupled to an RCA 8850 piotomultiplier, whose anode signal is transmitted to the diagnostics room for analysis, and the signal at the 10th dynode is preamplified for pulse height analysis on some shots. A transistor-regulated voltage divider ${ }^{\circ}$ is used to stabilize the dynode voltag distribution at high count rates and protect the PMT against excessive anode current. Anode pulses are analyzed by a pulse-shape discriminator so that an output is generated only for neutron induced events. In addjtion, a constant-fraction discriminator triggers this system and is used to set a lower energy threshold; thus, an output is generated only for recoil protons with greater than $1 \mathrm{MeV}$ of kinetic energy. This threshold provides substantial rejection of scattered neutron signals, while maintaining an adequately high overall efficiency.

Each recoil proton event at $E_{p}>1 \mathrm{MeV}$ generates a logic pulse that drives a resistor capacitor (RC) integration circuit. The integrator converts the recoil proton count rate to an analog signal whose amplitude is proportional to the neutron event rate and has a $100-\mu$ s time constant for responding to changes in yield. This anaiog signal is permanently recorded during a shot by the TMX physical data recorder.

\section{Calibration}

Neutron yield $\left(Y_{n}\right)$ from the TMX endplug is determined absolutely as a function of time, i.e., the detector count rate is measured and assuming a point, isotropic neutron source, the yield is estimated from Eq. (3).

$\mathrm{Y}_{\mathrm{n}}=\frac{4 \pi \mathrm{R}^{2} \mathrm{e}^{\Sigma X}}{\epsilon \alpha \eta} \mathrm{V}_{\text {out }}$,

where

$\mathbf{R}=$ distance from the monitor to plasma center ( $3.34 \mathrm{~m})$

$\Sigma=$ neutron attenuation coefficient for the diagnostic window at $2.45-\mathrm{MeV}$ neutron energy

$\mathbf{X}=$ thickness of the diagnostic window

E $=$ detector efficiency, counts per second resulting from unit neutron intensity $\left(1 \mathrm{n} / \mathrm{cm}^{2} \mathrm{sec}\right)$, determined for the energy threshold used in the experiment (Fig. 4)

$\alpha=$ conversion factor for the RC integrator circuit, $2.8 \times 10^{-7} \mathrm{~V} / \mathrm{cps}$

$\eta=$ correction factor for neutron background because of scattering. i.e., the ratio of total neutron intensity above system energy threshold to the unscattered intensity

$V_{\text {out }} \approx$ analog voltage at the output of the $R C$ integrator circuit.

Monitor efficiency, $\epsilon$, was determined at the LLL rotating target neutron source, RTNS-I. The system was exposed in its magnetic and electromagnetic shielding to neutrons produced at an angle of $100^{\circ}$ relative to the accelerated $400-\mathrm{keV}$ deuteron beam. At that angle the emitted neutron energy is $2.45 \mathrm{MeV}$ and the energy dispersion negligible. Neutron intensity was determined with an uncertainty of $\pm 5 \%$ using a calibrated proton recoil counter. Pulse height spectra were obtained from each of the two NE213 detectors and the counting efficiency, $\epsilon$, determined by integrating the pulse height spectra above various hypothetical proton energy thresholds. The results of these measurements are shown in Fig. 4, where the ratios of recoil proton count rate in the diagnostic monitor to the unscattered neutron intensity at the front face of the shield are plotted as a function of discriminator threshold energy. Threshold energy is determined by observing the detector pulse height from a ${ }^{22} \mathrm{Na}$ photon source permanently installed in the monitor. These data were corrected for a relatively small contribution from scattered neutrons in the RTNS-I environment by unfolding the pulse height spectra and determining the neutron energy spectrum at the detector. Uncertainties in the measurement of $\epsilon$ are estimated to be about $\pm 10 \%$, including errors in reproducing a particular energy threshold. 


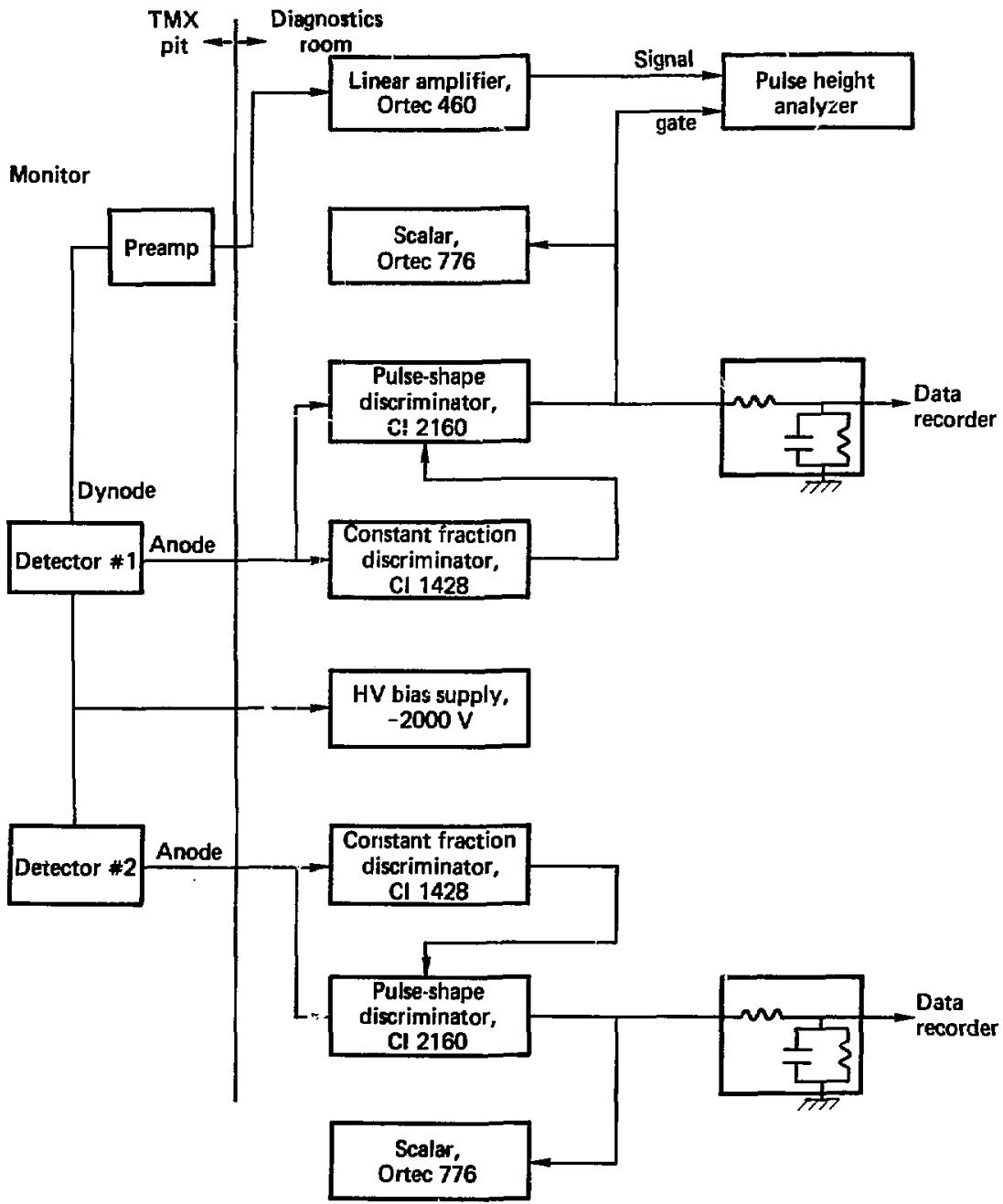

F]G. 3. Schematic of electronics configurations for TMX neutron monitor defectors and electrouics. The syatew sheme does not respend to spurious detector events caused by photons or by scattered neutrons at energies less than 1.0 MeV. 

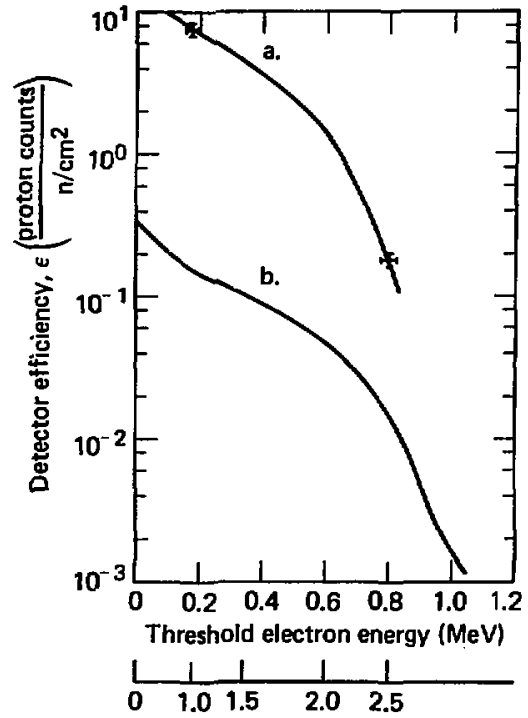

Threshold proton energy (MeV)

FIG. 4. Detector efficiency, $\epsilon=$ proton counts/ $\mathrm{n} / \mathrm{cm}^{2}$, plotted as function of lower energy threshold. Curve (a) is for the $5.1 \times 5.1 \mathrm{~cm}$ NE213 detector and (b) is tor the $1 \times 2 \mathrm{~cm} \mathrm{NE} 213$ detector. The two energy scales are shown on the abscissa. One is the proton (neutron) energy threshold; the other is the corresponding compton-electron energy, which produces the same amount of light in the scintillator. Error estimates are shown at two of the measured data points.

The relative intensity of scattered neutrons is greater near a TMX diagnostic window than at RTNS-I because of the close proximity of massive structures, including magnets, vacuum vessel walls, cryo-panels, and neutral beam injector ports. The relative contribution of this background to the diagnostic measurements was determined by measuring the neutron energy spectrum at the monitor on TMX. A pulse height spectrum was accumulated over a series of 34 shots at about $100 \mathrm{~A}$ of neutral beam current (Fig. 5). The data were unfolded using the L.LL unfolding code NUTSPEC ${ }^{7.8}$ to obtain the neutron energy spectrum at the monitor (Fig. 6). The spectral intensity above $1.0 \mathrm{MeV}$ of neutron energy was integrated, and compared to the integral from 2.2 to $2.6 \mathrm{MeV}$ to

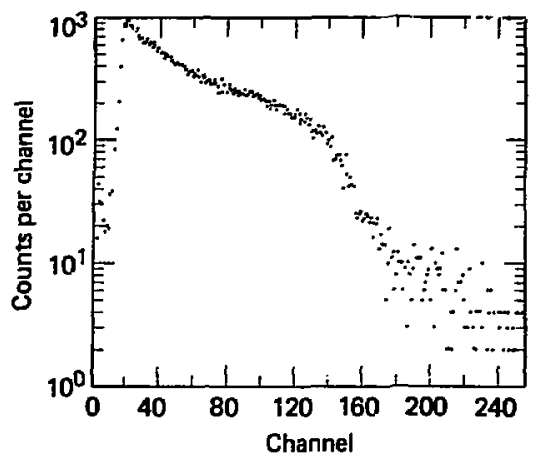

F1G. 5. Pulse height spectrum accumulated by the TMX neutron monitor during 34 discharges. The statistics are only marginally adequate for unfolding the neutron energy spectrum because of the fairly low yield of most of these shats $\left(\sim 0.5\right.$ to $\left.1.0 \times 10^{10} \mathrm{n} / \mathrm{s}\right)$.

determine that $\eta \approx 1.7$ at the TMX monitor. The uncertainty in that estimate is about \pm 0.15 . The measured value of $\eta$ agrees well with neutron transport calculations carried out in a somewhat similar geometry (MFTF) and with simple analytical estimates.

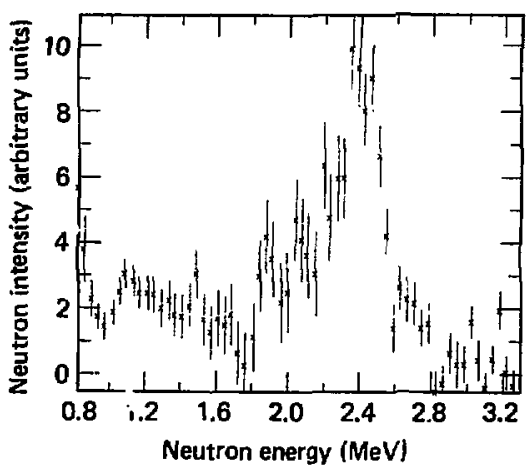

FIG. 6. Neutron energy specirum at TMX neutron monitor obtained by unfolding the pulse height data shown in Fig. 5. The resull shows the presence of significant scartered newtron intensily $\left(E_{w}<2.3\right.$ MeV) at the yield monitor. 
There are also background sources of neutrons resulting from the close proximity of neutral beam injectors. Deuterium builds up very rapidly in the injector ion dumps and in vacuum vessel walls, where it becomes a target for the accelerated deuterium. In the absence of target plasma, but with the magnets on, the average background neutron intensity at the monitor is equivalent to about $10^{10} \mathrm{n} / \mathrm{s}$ generated at plasma center when the $20-\mathrm{keV}$ neutral beam current is $\sim 100 \mathrm{~A}$. However, this background varies significantly from shot to shot and ranges from $2 \times 10^{9}$ to $2 \times 10^{10}$ at $I_{B}=100 \mathrm{~A}$. A similar measurement has not been made during highervoltage neutral beam injection.

Finally the conversion factor, $\alpha$, for determining pulse counting rate from an analog voltage, was measured using a photon source that produced a constant count rate. The circuit response is found to be linear up to about $5 \times 10^{5} \mathrm{cps}$. Above that rate there are errors not only caused by nonlinear response but also failures in pulse-shape discrimination that result in particle identification errors. Within the range of data reported here, $\alpha$ is known within about $\pm 5 \%$.

Uncertainties in determining absolute neutron yielõ are principally controlled by: 1) uncertainty in injector-produced background because individual beam currents vary from shut to shot even wher the beam-sum current is constant, 2) lack of information on the angular distribution of neutron emission from the plasma, and 3) nonreproducibility of a particular energy threshold for the monitor. Nuglecting the first two sources of error, the overall uncertainty in absolute neutron yield is estimated to be $\sim \pm 15 \%$.

\section{RESULTS}

A typical TMX discharge has a duration $\sim 25 \mathrm{~ms}$. In Fig. 7 the neutron yield in the east endplug is plotted for such a discharge. After stream gun operation, the neutron yield falls to an equilibrium leve' and remains there until the neutral beam injectors are turned off. Neutron yield was studied as a function of plasma line density, diamagnetic loop signal, and neutral beam current at equilibrium; the results are summarized below for the arbitrarily chosen time $t=16 \mathrm{~ms}$.

Generally, the neutron generation rate in the TMX endplug is in the range 0.8 to $3 \times 10^{11} \mathrm{n} / \mathrm{s}$ with $200 \mathrm{~A}$ of $17-\mathrm{keV}$ neutral beam injection. This is

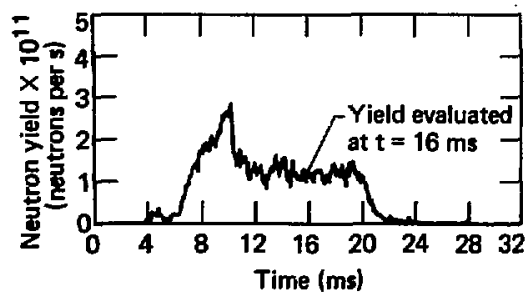

F1G. 7. Newtroi yield vo time for a typical east endying discharge. The yiekd date used to stedy scal-

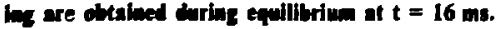

at least comparable to, and perhaps as much as, a factor of three higher than that observed in $2 \mathrm{X}$ and 2XIIB.9.10 In the discussions that follow, the neutron generation rate is correlated with plasma density, diamagnetism, and neutral beam current. of particular interest during this discussion is whether the majority of plasma fusion takes place within a single energetic ion component or there is evidence of reactions with a cold ion component.

\section{Scaling with Line Density}

Plasma line density is determined by measuring the attenuation of neutral beam current by the plasma. Neutron yield in the endplug varies with line density.

Data obtained up to now with 50 to $200 \mathrm{~A}$ of 20-keV deuterium injection into the endplug exhibits three distinct regimes. In the first, and most commonly observed regime, neutron yield is very widely scattered and uncorrelated with endplug density. These data are characteristic among shots during which center-cell parameters are varied. In the second, the neutron yield scales linearly with plasma line density; while in the third, the scaling is quadratic as observed in $2 \mathrm{XIIB}^{9,10}$ The latter behavior is most clearly observed in the data obtained during beam current scans on September 28, 
and December 13, 1979. Neutron yield at various line densities during those shots are shown in Fig. 8, together with a parabola that was least-squares fitted to the data. These data are consistent with a model in which the neutron yield results principally from hot ion reactions. On the other hand, a linear scaling of neutron yield with plasma density is frequently observed in the east endplug. In Fig. 9 is a typical example of this regime observed on August 7, 1979. This scaling is not well understood.

The observed scaling of neutron yield with density is partially explained in either of two ways, but neither is fully satisfactory. In the first it is assumed that neutron background resulting from injector ion dumps is important. The data in Figs. 8 and 9 are
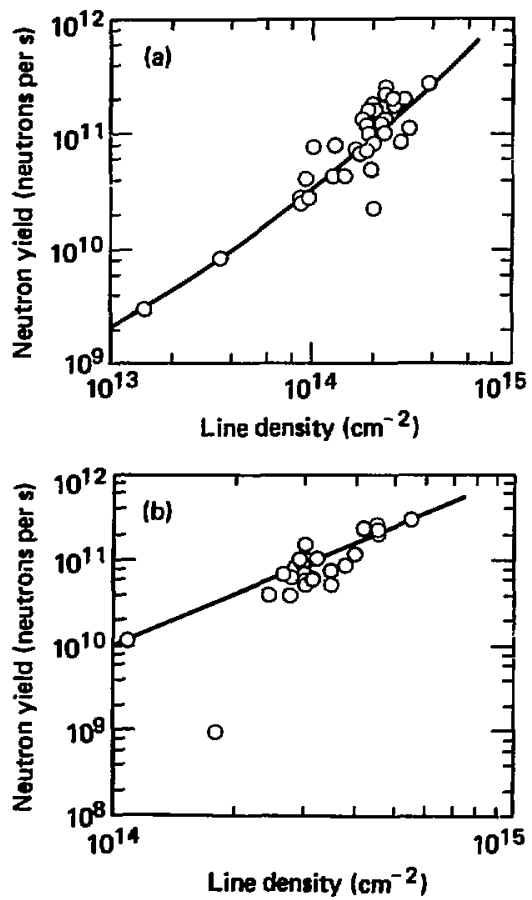

FIG. 8. Newtron yield vs plasma line density as the nentral bess current is varied. a) September 28 , 1979. Note the quadratic dependence of yield on density at $f$ inl $>1.5 \times 10^{14} \mathrm{~cm}^{-2}$. b) December 13 , 1979. These duta are fltted with a square law $Y_{n}=$ const $\left.X\left[\int n_{p} d\right]\right]^{2}$.

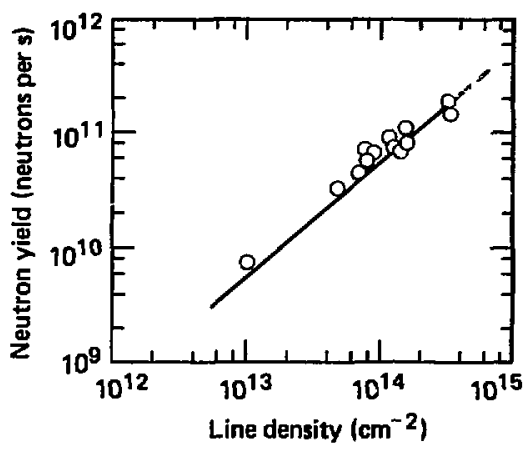

FIG. 9. Neutron yield vs pinsma line density on August 7, 1979. Note the linear dependence.

not compcnsated for this background, because that contribution on a given shot is only known within a factor of ten. Neutron background would be expected to vary linearly with beam current if the principal source was reactions between accelerated del ${ }^{+}$sicns and deuterium implanted in walls and beam dumps. Because the density also varies linearly with beam current at $t=16 \mathrm{~ms}$, the observed linear scaling of yield with density would seem to be explained in the low density regime, where this background is most important. However, the data in Fig. 9 show that the linear regime may persist up to high densities where the observed neutron yield is more than ten times higher than the largest background so far recorded. In addition, the neutron yield on August 7th scaled linearly with the diamagnetic loop signal (Fig. 10). Finally, the

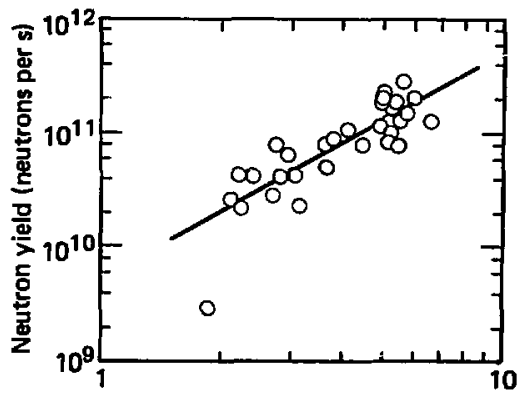

Diamagnetic loop signal (arbitrary units)

FIC: 10. Newtron yield vs diamaguetic loop sigeal on Auguat 7, 1979. The solid lise is a lenst-squares IIt of a linear function to the data. 
neutron yield decays with a characteristic time of $\tau \approx 600 \mu \mathrm{s}$ following beam shut-off. The background contribution should fall with a time constant of $\tau \approx 100 \mu$ s corresponding to the instrument time-response, because the beam shut-off time is only about $10 \mu \mathrm{s}$.

An alternative explanation assumes a twocomponent plasma containing hot ior: and cold ions. The latter group may arise because center-cell plasma penetrates the endplug. The neutron yield would then be described by Eq. (4).

$Y_{n}=\left[\frac{1}{2} n_{i: H}^{a}\langle\sigma v\rangle_{H}+n_{H} n_{C}\langle\sigma v\rangle_{H C}+\frac{1}{2} n_{C}^{2}\langle\sigma v)_{C}\right] V$

Because the cross sections are rapidly rising functions of ion energy, the third term probably can be neglected. The scaling derived from Eq. (4) depends on the ratio $n_{H} / n_{C}$. The cross section data in Miley et al. ${ }^{2}$ indicates that for $W_{i h_{0}} \approx 10 \mathrm{keV}$ and $W_{\mathrm{j} \text { cold }}<1 \mathrm{keV}$, the second term is important only for $\mathrm{n}_{\mathrm{H}} / \mathrm{n}_{\mathrm{C}}<0.01$.

The model described above is, in principle, adequate to explain the large variation in $Y_{n}$ observed when center-cell parameters are varied and may be consistent with an occasional linear relation between $Y_{n}$ and the endplug diamagnetism. It nevertheless seems to fail three important tests. First, electrostatic confinement of center-cell plasma by the endplug would not be good for $n_{\mathrm{H}} / \mathbf{n}_{\mathrm{C}}<0.01$ in the endplug. Second, the abscissa in Figs. 8 and 9 are total line density, i.e., $\int\left(n_{H}+n_{C}\right) d l$, that in the region of interest here, is dominated by $\mathrm{n}_{\mathrm{C}}$. Third, such a cold plasma is in total disagreement with measured plasma diamagnetism in the endplug. Thus, the observed scaling of $Y_{n}$ with endplug density must be considered poorly understood. It appears that centercell parameters may have a considerable impact on the fusion rate in the endplug.

\section{Scaling with Plasina Dianagnetism}

There are two diamagnetic loops on the TMX east endplug. The variation of neutron yield with the mean signal obtained on these loops has been studied at $\mathrm{t}=16 \mathrm{~ms}$. A plot of the results obtained on September 28, 1979 (corresponding to the data in Fig. 8a) is shown in Fig. 11. The figure includes a line showing a least-squares fit of a function proportional to the square of measured djamagnetism. A

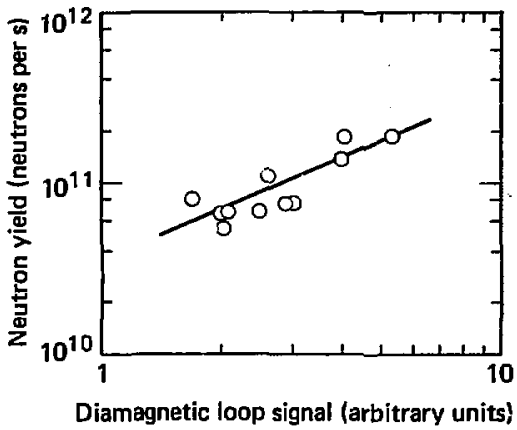

FIG. il. Neutron yield rs diamagnetic loop signal on September 28, 1979. The solid line is a leastsquares fit of a square law function to the data.

square-law relation between neutron yield and plasma diamagnetism is well established. On the other hand, in the data obtained on August 7, 1979, where yield scaled linearly with density (Fig. 10), the results follow a linear function of plasma diamagnetism that is shown in the figure.

\section{Scaling with Neutral}

\section{Beam Current}

During the experiments reported here, the TMX endplug was heated using 50 to $220 \mathrm{~A}$ of neutral deuterium at 16 to $17 \mathrm{keV}$ obtained from the 20-kV neutral beam injectors. Some discharges included up to 10 to $15 \mathrm{~A}$ from the $40-\mathrm{kV}$ injectors, but that contribution to the neutron yield was not studied separately. Most of the data obtained exhibit a square-law dependence of neutron yield on beam current, including the data obtained on both September 28, and August 7, 1979. In Fig. 12 is a typical set of data, obtained during the beam current scan on September 28, 1979. The line in the figure is a least-squares fit of a square-law function to the data.

\section{Decay of the Fusion Rate Following Beam Shutoff}

Plasma cooli..g and the decay of plasma density following terminating neutral beam injection causes a rapid decay of the neutron generation rate. Analysis of this decay may be helpful in establishing the relative importance of hot-hot and hot-cold or beam-target reactions because the latter would be expected to drop nearly stepwise after the beam is 


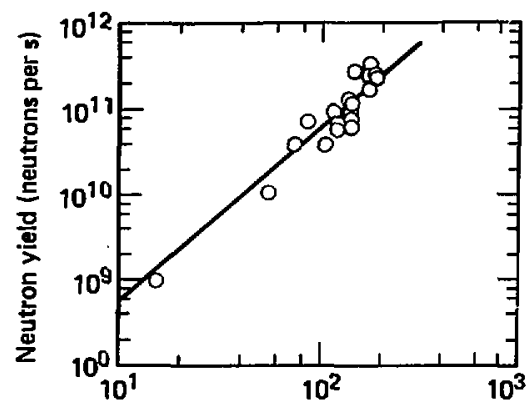

Total neutral beam current at $20 \mathrm{kV}$ (A)

FIG. 12. Neutron yield ws total neutral beam current at $20 \mathrm{keV}$, December 13, 1979. The solid curve is a square law function fitted to the data.

terminated. A typical plot of neutron yield vs time after terminating injection is placed in Fig. 13 along with a plot of plasma line-density. Two features are important. First, there is no precipitous drop in neutron yield at the time of beam shut-off, as would be the case for a predominantly beam-target source. Second, the time constant for the decay of neutron generation is about $0.6 \mathrm{~ms}$ in all of the shots studied. That decay rate is more than twice the density decay ( 1.5 to $2.5 \mathrm{~ms}$ ) and is consistent with a hot-hot reaction source. The same decay rate was observed for neutron yield in $2 \mathrm{X} 11 \mathrm{~B},{ }^{10}$ although the density decay there was not as fast.

The ion-energy decay time, $\tau_{E}$, may be determined from the neutron-generation decay rate and the density decay. This is accomplished as follows:

The neutron yield is assumed to arise principally from hot-hot reactions, and Eq. (4) reduces to

$Y_{n}=\frac{1}{2} n_{H}^{2}\{\sigma v\rangle V$.

Then, if it is assumed that the reaction cross section varies as $\sigma=\sigma_{0} W_{i}^{\alpha}$,

If the plasma density decays exponentially with the time constant $\tau_{p}$, then the neutron yield decays according to

$$
Y_{n}=\frac{1}{2} n_{0}^{2} e^{-2 t / \tau_{p}}\left\langle\sigma_{0} v_{0}\right\rangle\left[\frac{\overline{W_{i}}(t)}{\overline{W_{i}(0)}}\right]^{\alpha+1 / 2} v .
$$

If Fq. (7) is rewritten in terms of the ion energy decay time, $\tau_{E}$, then

$$
Y_{n}=\frac{1}{2} n_{0}^{2} \mathrm{e}^{-2 t / \tau_{\mathrm{p}}}\left\langle\sigma_{0} v_{0}\right\rangle \mathrm{e}^{-(\alpha+1 / 2) t / \tau_{\mathrm{E}}} \mathrm{V},
$$

where $\tau_{E}$ is defined by

$$
\bar{W}_{j}(t)=\bar{W}_{j}(0) e^{-t / \tau} E
$$

The neutron gield decay time, $\tau_{\mathrm{N}}$, is then related to $\tau_{p}$ and $\tau_{E} b y$

$\frac{1}{\tau_{N}}=\frac{2}{\tau_{p}}+\frac{\alpha+\frac{1}{2}}{\tau_{F}}$.

Analysis of the data shown in Fig. 13 shows that $\tau_{\mathrm{N}}=0.54 \mathrm{~ms}$, and $\tau_{\mathrm{p}} \sim 2 \mathrm{~ms}$. The D-D reaction cross-section dat ${ }^{2}$ in the range 10 to $20 \mathrm{keV}$ suggest values for $\alpha$ in the range 2 to 5, depending on the angular distribution of reacting ions. Reasonable assumptions regarding this distribution reduces the probable range to between 3 and 4 . It follows that the ion energy-decay time, $\tau_{\mathrm{E}}$, in the shots studied is in the range $\tau_{E} \sim 4$ to $6 \mathrm{~ms}$. This estimate is consistent with $\tau_{\mathrm{drag}}=5.8 \mathrm{~ms}$ based on electron drag cooling at $\mathrm{n}_{\mathrm{e}}=1.2 \times 10^{13} \mathrm{~cm}^{-3}$ and $\mathrm{T}_{\varepsilon}=137 \mathrm{eV}$, the parameters corresponding to the shot from which the data in Fig. 13 were obtained.

\section{ESTIMATING MEAN ION ENERGY $\bar{W}_{\mathrm{i}}$}

The mean energy of the hot ions may be estimated from the neutron yield, provided that the measured yield results principally from reactions among these jons. The data obtained at high yield on September 28th and the yield decay data shown in Fig. 13 seem to warrant such a presumption. 


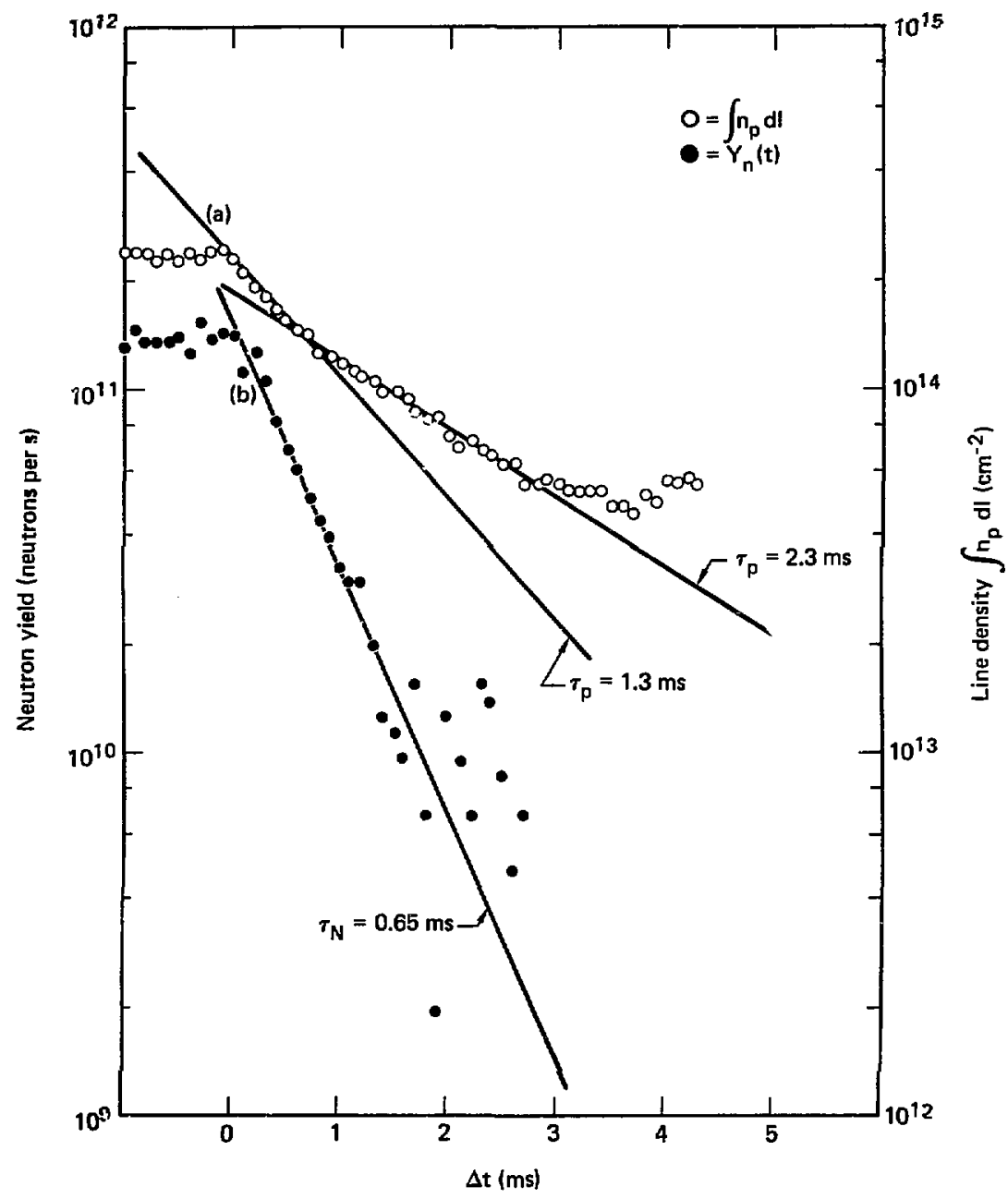

F1G. 13. Decay of line density and neutron yield following termination of neutral beam injection; a) line density, b) meatron yiek. Note the absence of a sharp drop in neutron yield at the time of beam termination $(\Delta t=0)$ and the exponential decay in yield as the plasma density falls following beam termination. Lines shown are fits of an exponemtial function through the data. Time constants shown are the respective 1/e decay times, 
Several shots in this regime were evaluated. The plasma volume is assumed to be an ellipsoid of radius $R_{p}=8 \mathrm{~cm}$ and half-length $L_{p}=18 \mathrm{~cm}$, with a gaussian density profile in $r$ and $Z$ given by Eq. (11).

$$
n_{H}(r, Z)=n_{0} e^{-\left(r / R_{p}\right)^{2}-\left(Z / L_{p}\right)^{2}}
$$

The radial line density, $\int \mathrm{n}_{\mathrm{p}} \mathrm{dl}$, and effective plasma volume, V, are evaluated from Eq. (11) and then combined with Eq. (5), to obtain an expression of $\langle\sigma v\rangle$ from the neutron yield and line density measurement. The result is

$$
\langle\sigma v\rangle=\frac{4 \sqrt{2} Y_{n}}{\sqrt{\pi} L_{p}\left[\int n_{p} d\right]^{2}} .
$$

Equation (12) provides a spatial average of $\langle\sigma v\rangle$, weighted by the fartor $n^{2} \mathrm{H}(r, Z)$. As a result, it is primarily sensitive .0 the central-plasma ionenergy distribution. The reaction cross section, $\sigma$, is very sensitive to ion ent:gy so that $\langle\sigma v\rangle$ is a very sensitive measure of $\bar{W}_{\mathrm{i}}$. Lehner ${ }^{11}$ presents calcula- tions for several different ion energy distributions ranging from maxwellian to monoenergetic. It is apparent from those calculations that $\langle\sigma v\rangle$ may vary by a factor of 2 to 3 among different assumed energy distributions and by the same factor for head-on vs isotropic ion trajectories, but these variations are small compared to those from small changes in $\bar{W}_{\mathrm{j}}$. As a result, the mean energy may be determined with relatively high precision in spite of large uncertainties in the energy and angular distributions or even in $\langle c\rangle$.

Measurements made for several shots, on September 28, 1979 are summarized in Table 1. Although the densities vary by at least a factor of two (within the square-law regime of Fig. 8) and the yields vary by a factor of more than three, $\langle\sigma v\rangle$ inferred from these measurements is remarkably constant. The ion energy, $\bar{W}_{i}$ inferred from Lehner's calculations is relatively independent of the form assumed for the ion energy distribution and is consistent with the neutral-beam injection energy of $\sim 16$ to $17 \mathrm{keV}$. These energies are also very similar to those determined from ion-energy spectrum measurements on 2 XIIB. 9

\begin{tabular}{|c|c|c|c|c|c|c|}
\hline Date & Shot $\mathrm{Na}$ & $\underset{\mathrm{cm}^{-3}}{\mathrm{~cm}_{\mathrm{p}} \mathrm{dl},}$ & $\begin{array}{l}Y_{n}, \\
n / s\end{array}$ & $\begin{array}{l}(\sigma \mathrm{v}) \\
\mathrm{cm}^{3} / \mathrm{s}\end{array}$ & $\begin{array}{l}\bar{w}_{\mathrm{i}^{\prime}}{ }^{2} \\
\mathrm{keV}\end{array}$ & $\begin{array}{l}\overline{\mathbf{w}}_{\mathbf{i}}^{\mathbf{b}} \mathbf{b} \\
\mathrm{keV}\end{array}$ \\
\hline \multirow[t]{3}{*}{ September 28, 1979} & 22 & $3.89 \times 10^{14}$ & $2.9 \times 10^{11}$ & $3.5 \times 10^{-19}$ & 10.3 & 12.7 \\
\hline & 23 & $29 \times 10^{14}$ & $2.0 \times 10^{11}$ & $4.2 \times 10^{-19}$ & 11.3 & 13.8 \\
\hline & 47 & $20 \times 10^{14}$ & $8.3 \times 10^{10}$ & $3.8 \times 10^{-19}$ & 10.5 & 13.2 \\
\hline October 28, 1979 & 6 & $23 \times 10^{14}$ & $1.4 \times 10^{1 !}$ & $4.7 \times 10^{-19}$ & 11.6 & 14.2 \\
\hline
\end{tabular}

TABLE 1. Mean ion energy, $\overline{\mathrm{W}}_{\mathrm{i}}$, estimated from neutron yield.

\section{CONCLUSION}

Neutron yield measurements on the TMX east endplug show generation rates up to $\sim 3 \times 10^{11} \mathrm{n} / \mathrm{s}$ with $200 \mathrm{~A}$ of neutral deuterium at 16 to $17 \mathrm{keV}$. This is comparable to, or slightly higher than, neutron generation rates in 2 XIIB at the same beam current. There appear to be two distinct regimes of operation. One regime exhibits a neutron generation rate that scales linearly with plasma density and diamagnetism, while in the other the scaling is a square-law. The former regime is something of a surprise and is not well understood, although the data there may be consistent with a two-component 
plasma containing cold ions among the hot plasma jons formed by charge exchange with the neutral beam. The square-law regime was observed in $2 \mathrm{X}$ and $2 X I I B$, the neutron generation rate scales with other parameters according to expectations for reactions among hot ions.

Measurements of the decay of neutron ge. tera- tion following beam shut-off were used to determine the ion-energy decay time, $\tau_{E}$, and the results are in good agreement with electron-drag cooling of the plasma. Similarly, neutron yield miasurements were used to estimate the mean ion energy and the results are consistent with a plasma produced by chargeexchange trapping of the injected neutral beams.

\section{ACKNOWLEDGMENTS}

The author wishes to thank members of the TMX physics staff for their continued interest, help, and constructive suggestions. Special thanks go to T. Simonen, D. Grubb, and A. Hunt for their support and encouragement.

\section{REFERENCES}

1. F.H. Coensgen, TMX Major Project Proposal, Lawrence Livermore Laboratory, Livermore, Calif., LLL-Prop-148 (1977).

2. G.H. Miley, H. Towner, and N. Ivich, Fusion Cross Sections and Reactivities, University of Illinois, Urbana, Ill., COO-2218-17 (1974).

3. J. Kirkbride, Nucl. Instrum. Methods 52, 293 (1967).

4. F.T. Kuchnir and F.J. Lynch, IEEE Trans. Nucl. Sci. NS-15 (3), 107 (1968).

5. V.V. Verbinski, W.R. Burrus, T.A. Love, W. Zobel, and N.W. Hill, Nucl. Instrum. Methods 65,8 (1968).

6. D.R. Slaughter, IEEE Trans. Nucl. Sci, NS-26 (1), 802 (1979).

7. D.R. Slaughter, D.W. Rueppel, ard D.A. Fuess, Neutron Spectrum Measurements for Radiation Protecfion Purposes, Lawrence Livermore Laboratory, Livermore, Calif., UCRL-52415 (1978).

8. D.R. Slaughter, NUTSPEC: A Program for Unfolding Neutron Spectral Data Obtained With Scintillation Detectors and Gas Proportional Counters, Lawrence Livermore Laboratory, Livermore, Calif., UCID17713 (1978).

9. F.H. Coengen, W.F. Cummins, R.E. Ellis, and W.E. Nexsen, "Decay Modes of a Dense Plasma in a Magnetic Well," Intemational Atomic Energy Agency, 1968 (Novosibirsk, USSR 1968) paper $\mathrm{CN}-24 / \mathrm{H}-2$.

10. T.C. Simonen, Lawrence Livermore Laboratory, Livermore, Calif., private communication (1979).

11. G. Lehner, "Nuclear Reactions in Fully Ionized Plasmas at High Temperature." in Reactions Under Plasma Conditions, Vol. II, M. Venugopalan, Ed. (Wiley-Interscience, N.Y., 1971), p. 519. 